ISSN: $1130-3743$

\title{
ESTILOS EDUCATIVOS PARENTALES. REVISIÓN BIBLIOGRÁFICA Y REFORMULACIÓN TEÓRICA
}

\section{Parenting styles. Bibliographical revision and theoretical reformulation}

\author{
Modèles éducatifs parentales. Révision bibliographique \\ et reformulation théorique
}

\begin{abstract}
Susana Torío López, José Vicente Peña Calvo y M. a del Carmen Rodríguez Menéndez Universidad de Oviedo. Facultad de Ciencias de la Educación. Departamento de Ciencias de la Educación. C/ Aniceto Sela, s/n. 33005 Oviedo. Correo-e: storio@uniovi.es; vipe@uniovi.es; carmenrm@uniovi.es
\end{abstract}

Fecha de recepción: enero de 2008

Fecha de aceptación definitiva: abril de 2008

BIBLID [(1130-3743) 20, 2008, 151-178]

RESUMEN

Los padres, en relación con los hijos, son los modelos de referencia más importantes de su vida y la falta de apoyo y de responsabilidad parental son actos que ocasionan graves consecuencias para un desarrollo equilibrado. Desde los estudios emprendidos por Baumrind, investigadora que durante más de treinta años ha estudiado las tipologías de estilos disciplinares y el comportamiento infantil, la literatura psico-pedagógica producida en esta materia ha sido abundante, por ser uno de los elementos claves de la socialización familiar. El objetivo de estas páginas es revisar las investigaciones que relacionan los estilos educativos paternos y el desarrollo de la infancia y la adolescencia, con el fin de realizar una propuesta integradora que permita el desarrollo de programas de intervención socioeducativa.

Palabras clave: familia, estilos educativos familiares, educación familiar, disciplina, socialización infantil y adolescente. 


\section{SUMMARY}

Parents constitute the most important life model for children. Moreover, the absence of their support and the lack of parental responsibility lead to serious consequences for children's balanced development. Beginning with the research carried out by Baumrind, who devoted over thirty years to studying the typologies of disciplinary styles and child behaviour, the psycho-pedagogical literature about these important topics in family socialization has been profuse. The aim of this paper is to review the investigations which relate parenting styles to childhood and adolescence, in order to offer an integrated proposal which can make the development of socioeducative programs of intervention feasible.

Key words: family, parenting styles, parent's education, discipline, childhood and adolescence socialization.

\section{SOMMAIRE}

Par rapport aux enfants, les parents sont les modèles référentiels les plus importants de leur vie et le manque de soutien et de responsabilité parentale sont des actes ayant de graves conséquences sur un développement équilibré. Depuis les études entreprises par Mme. Baumrind, chercheur qui a étudié les typologies des styles disciplinaires pendant plus de trente ans, la littérature psychopédagogique produite dans cette matière est abondante, étant donné qu'il s'agit d'un élément clé de la socialisation familiale. À partir de l'analyse des recherches qui rattachent les styles éducatifs paternels et le développement de l'enfance et de l'adolescence, le but de ce travail est de réaliser une proposition intégrative permettant le développement de programmes d'intervention.

Mots clef: famille, modèles éducatifs familiaux, éducation familiale, discipline, socialisation des enfants et des adolescents.

\section{INTRODUCCIÓN}

Nadie parece poner en duda que la familia es el contexto de crianza más importante en los primeros años de vida, adquiriendo en él, niños y niñas, las primeras habilidades (reír, hablar, jugar) y los primeros hábitos que le permitirán conquistar su autonomía y las conductas cruciales para la vida. Los adultos que los cuidan tienen un importante papel en la vida de los niños pero, aunque importante, muchas veces dicha influencia no es la decisiva, no aprenden solamente de ellos. Podemos decir que "la educación no es algo que los padres hagan a los hijos, sino algo que padres e hijos hacen conjuntamente» (Rich, 2002, 53) ${ }^{1}$.

1. La autora Rich Harris, en su libro El mito de la educación, muy citado en la bibliografía sobre familia, cuestiona la concepción tradicional de la crianza y educación de los hijos. Los investigadores 
En las páginas que siguen, revisamos las investigaciones que relacionan los estilos educativos familiares y el desarrollo y bienestar de la infancia y de la adolescencia. En primer lugar, desde nuestra perspectiva, nos parece importante realizar esta revisión por la existencia de conceptualizaciones diversas y la necesidad de una sistematización de tal problemática. En segundo lugar, por la importancia que presentan los aprendizajes adquiridos en el ámbito familiar: "Los hogares, los espacios privados, y las relaciones íntimas, familiares, facilitan comportamientos que sólo aquí pueden fructificar provechosamente, o malograrse trágicamente. En realidad, estos espacios o relaciones son los semilleros de la estimativa humana" (Rodríguez, 1999, 86). En el momento actual, ser padre o madre es una tarea complicada en la que no sirve la improvisación y se exigen destrezas específicas ante las nuevas necesidades que surgen en la sociedad. Los estilos educativos representan la forma de actuar de los adultos respecto a los niños ante situaciones cotidianas, la toma de decisiones o la resolución de conflictos. Por tanto, se ponen en juego unas expectativas y modelos, se pretende regular las conductas y marcar unos parámetros que serán el referente tanto para comportamientos como actitudes.

Por último, otro motivo que nos ha llevado a la elección de esta temática es la necesidad de profundización en el proceso de intervención socioeducativa en el ámbito familiar. Hoy en día no existe duda de la necesidad de desarrollar programas de educación familiar y conllevan cambios en la forma de educar, en las prácticas disciplinarias y en la atención que los padres prestan a los hijos.

\section{DiMENSIÓN FORMAL DE LA SOCIALIZACIÓN FAMILIAR}

Para el estudio de la socialización del niño, en el seno de la familia, se han combinado diferentes enfoques teóricos (psicoanalítico, teorías del aprendizaje, enfoque humanista, etc.), surgiendo diversos modelos en los que se tienen en cuenta variables tales como el control, la comunicación o la implicación afectiva entre los miembros de la familia. De la interrelación de las variables que se producen en las prácticas educativas parentales surgen los diferentes estilos educativos. Vamos a detenernos, en primer lugar, en analizar las estrategias de socialización o variables que algunos autores destacan en el proceso de socialización para, a

\footnotetext{
de la socialización mantienen que lo que los niños aprenden en esa temprana edad acerca de las relaciones y las reglas establece el modelo para posteriores relaciones y, por lo tanto, determina el curso de sus vidas. Para la autora lo que han demostrado estas investigaciones es que "la conducta de los padres hacia un hijo afecta sobre todo a cómo se comporta el hijo en presencia de los padres o en contextos que están asociados con ellos" (p. 116). Es el entorno de fuera del hogar lo que puede cambiar al niño, no la crianza. La alternativa que presenta es la "Teoría de la socialización grupal", no son los padres quienes socializan a sus hijos, son los propios niños los que se socializan entre ellos, el grupo dentro del cual el niño se relaciona con sus iguales, el entorno fuera del hogar y el que comparte con sus compañeros: "no todo tiene que ver con la socialización, sino también con el modo como las personalidades de los niños se moldean y cambian por las experiencias que tienen mientras crecen" (p. 199).
} 
continuación, desarrollar los modelos o estilos que se conforman a partir de dichas estrategias socializadoras.

\subsection{Estrategias y mecanismos de socialización familiar}

El conjunto de conductas que los padres valoran como apropiadas y deseables para sus hijos, tanto para su desarrollo como para su integración social, reciben el nombre de estrategias de socialización, lo que los padres desean que ocurra respecto a sus hijos y los medios para alcanzar esos estados deseables (Goodnow, 1985). Estas metas y estrategias de socialización que emplean los padres con los hijos tienen que ver con el tono de la relación, con el mayor o menor nivel de comunicación (aceptación-rechazo, calor-frialdad, afecto-hostilidad, proximidaddistanciamiento) y con conductas para encauzar el comportamiento del niño o la niña (autonomía-control, flexibilidad-rigidez, permisividad-restricción). Son diversas las variables fundamentales o dimensiones que se combinan y se han formulado para dar como resultado unos estilos educativos determinados.

Desde mediados del siglo pasado se han venido identificando en las relaciones padres-hijos al menos dos variables en las prácticas educativas parentales, consideradas fundamentales en la socialización de los hijos, variables denominadas como "dominio-sumisión" y "control-rechazo", y que Rollins y Thomas (1979) las definen como "intentos de control" y "apoyo parental". Gran parte de la literatura que aborda la interacción familiar se refiere a la descripción de estas dos dimensiones (Erikson, 1963; Hoffman, 1975a; Baumrind, 1991a; Flaquer, 1993; Musitu, Román y Gutiérrez, 1996; Molpereces, Llinares y Musitu, 2001; Gadeyne, Ghesquière y Onghena, 2004).

Todos estos autores constatan la variedad de pautas que pueden emplear los padres en las estrategias de socialización de sus hijos basadas en las dimensiones de apoyo y control parental. Por ejemplo, cuando un niño o niña se porta mal, algunos padres opinan que lo más educativo es retirarle un privilegio (no ver la televisión o no salir a jugar); otros opinan que lo mejor es sentarse a hablar con ellos y analizar su comportamiento; otros pueden opinar que lo mejor es dejar a los hijos a "su aire" para que aprendan por sí mismos. Sin duda, todos los padres desean lo mejor para sus hijos, pero lo importante es conocer el modo en que aparecen las distintas estrategias educativas, de acuerdo a condicionamientos como la edad, la situación en particular, o la madurez psicológica del niño en cada momento educativo.

Dichas dimensiones se han disgregado con el paso del tiempo y, en la actualidad, se consideran cuatro aspectos distintos en las conductas de los progenitores: afecto en la relación, el grado de control, el grado de madurez y la comunicación entre padres-hijos (Moreno y Cubero, 1990; Solé, 1998; Ochaita, 1995). Estas dimensiones y su combinación procuran unas experiencias educativas diversas que los niños viven en su familia y que naturalmente influirán en su desarrollo. 
En este sentido, el apoyo se define como conducta expresada por un padre o la madre hacia un hijo, que hace que el niño se sienta confortable en presencia del mismo y confirme, en la mente del niño, que es básicamente aceptado como persona (Thomas, Gecas, Weigert y Rooney, 1974; Rollins y Thomas, 1979). Esta variable ha tenido gran número de etiquetas relativamente similares: aceptación, educación o amor y conductas parentales como las alabanzas, elogios, aprobación, estimulación-aliento, ayuda, cooperación, expresión de términos cariñosos, ternura y el afecto físico. Estas conductas son consideradas como "sanciones sociales positivas difusas" por los sociólogos y antropólogos sociales, como "estímulos reforzantes positivos" por los psicólogos del aprendizaje y como "caricias positivas" por los psicólogos transaccionales (Musitu, Román y Gracia, 1988, 105).

Por otra parte, el intento de control se define como la conducta de un padre hacia un hijo con el objeto de dirigir la acción de éste de una manera deseable para los padres (Musitu, Román y Gracia, 1988, 106). Esta concepción es coincidente con la disciplina familiar y se utilizan términos como dominancia, restricción o coerción. Es una dimensión crucial en el desarrollo de la persona, puesto que a través de la guía y el control que ejercen los otros aprendemos a regular y controlar nuestra conducta de manera autónoma.

El grado de madurez se relaciona con los retos y exigencias que los progenitores imponen a sus criaturas. La capacidad para establecer un ambiente comunicativo es otra de las dimensiones en que las prácticas educativas se distinguen. Dicha dimensión se refiere a la posibilidad de crear una dinámica en la que es posible explicar de manera razonada las normas y las decisiones que se toman teniendo en cuenta el punto de vista de los otros. Permite compartir problemas, conflictos, dudas, satisfacciones, etc.

Estas variables, junto con el poder, que se define como el potencial que un individuo tiene para obligar a otra persona a actuar de modo contrario a sus propios deseos, serán utilizadas para explicar las conductas socialmente competentes e incompetentes de los niños.

Por otra parte, Schwarz, Barton-Henry y Pruzinsky (1985) han definido las prácticas de socialización familiar en tres dimensiones o ejes fundamentales:

a) Una dimensión de aceptación que abarcaría desde la implicación positiva, el centrarse en el hijo hasta el rechazo y la separación hostil.

b) Una dimensión de control firme que implica grados diferentes tales como el refuerzo, la falta de refuerzo, la disciplina laxa o la autonomía extrema.

c) Una dimensión de control psicológico en la que se incluyen grados como la intrusión, el control hostil, la posesividad y la retirada de la relación.

Asimismo, Coloma (1993a, 48) destaca las variables siguientes, enmarcadas en dos polos opuestos: a) control firme en contraposición a control laxo; b) cuidado y empatía en contraposición a rechazo e indiferencia; c) calor afectivo en contraposición a frialdad-hostilidad; d) disponibilidad de los padres a responder a las señales de los hijos en contraposición a la no disponibilidad; e) comunicación 
paternofilial bidireccional frente a una comunicación paternofilial unidireccional; f) comunicación paternofilial abierta frente a la cerrada, etc.

\subsection{Estilos educativos parentales y consecuencias educativas sobre la socialización de los hijos: principales investigaciones}

Los modelos o estilos educativos se configuran a partir de las variables anteriormente descritas y en función del peso que cada una de ellas tiene en el entramado de las relaciones padres e hijos. Desde esta perspectiva, podemos definir los "estilos educativos parentales" como: "esquemas prácticos que reducen las múltiples y minuciosas prácticas educativas paternas a unas pocas dimensiones, que, cruzadas entre sí en diferentes combinaciones, dan lugar a diversos tipos habituales de educación familiar" (Coloma, 1993a, 48).

En el ámbito de la sociología y psicología social, son bastante numerosos los trabajos sobre los estilos educativos de los padres y su influencia en el desarrollo infantil. No se identifican modelos puros, sino que se solapan, ya que los estilos educativos suelen ser mixtos y varían con el desarrollo del niño, no siendo estables a lo largo del tiempo. Además, pueden cambiar de acuerdo a múltiples variables, como son: el sexo, edad, lugar que ocupa entre los hermanos, etc. Por ello, es preciso analizar los estilos educativos en el contexto de los cambios sociales, valores predominantes, realidad de cada familia o en el contexto del momento evolutivo en que se encuentre el niño o la niña. En palabras de Rich Harris (2002, 53): "... los padres no tienen un estilo educativo fijo. El modo como se comporta un padre respecto de un niño en particular depende de la edad del niño, de su apariencia física, de su conducta habitual, de su conducta pasada, su inteligencia y su estado de salud. Los padres confeccionan su estilo educativo a medida de cada niño". Por tanto, cuando se habla de estilos de prácticas educativas parentales, nos referimos a tendencias globales de comportamiento. Más importante que saber si unos padres/madres son de un tipo u otro, es tener conocimiento sobre las dimensiones presentes en una interacción de calidad. En este sentido, percatarse que la comunicación y el afecto no están reñidos con la exigencia y el control.

Somos conscientes de la simplicidad de algunos modelos al ser descritos, pero hay que resaltar que las relaciones padres-hijos son bidireccionales. Lejos de mantener una postura pasiva por parte del niño, se entiende que los hijos también influyen sobre los padres de modo decisivo. Podemos definir un modelo de relación padres-hijos como: "un sistema organizado de actuaciones, creencias y actitudes que implican conductas diversas" (Torres, Alvira, Blanco y Sandi, 1994, 23).

Dentro de este ámbito de investigación, uno de los modelos más elaborados es el de Diana Baumrind, trabajo clásico de los años 1967 y 1971, en el que se tiene en cuenta la interrelación entre las tres variables paternas básicas: control, comunicación e implicación afectiva. El trabajo de dicha autora recibe influencias de Kurt Lewin y su equipo de investigación, quienes aplicaron su teoría al ámbito de la 
familia y el matrimonio. Lewin reconocía la importancia de la familia como un campo interpersonal y reflexionó sobre aspectos como el orden de los hermanos, la composición familiar y la interdependencia de los esposos. Sus trabajos influyeron en Diana Baumrind quien, continuando la tradición lewiniana del estudio de los grupos, escogió, como tema principal de su tesis doctoral, la autoridad en los grupos de discusión. Pero Diana Baumrind se hizo famosa por el estudio de la autoridad en el ámbito de las relaciones padres-hijos, convirtiéndose en una pionera en el estudio de los estilos parentales de socialización. Al igual que Kurt Lewin, Diana Baumrind reconocía los déficits del control autoritario.

Esta autora ha llevado a cabo investigaciones sobre socialización familiar durante los últimos treinta años y su trabajo representa un punto de referencia fundamental en el campo del apoyo y control parental, la autonomía y el desarrollo del hijo. Baumrind (1966, 1967, 1968, 1971, 1972, 1978, 1991a, 1991b, 1996, 1997) pretende descubrir si determinados estilos educativos de los padres correlacionan con el desarrollo de cualidades y características en el niño. En estos trabajos, más que estudiar dimensiones aisladas, se consideran grupos de características que suelen aparecer simultáneamente.

Diana Baumrind realiza su investigación con 134 niños y niñas, menores de tres años, que estaban escolarizados. Las madres y los padres eran entrevistados y, a la vez, se observaba la conducta que empleaban éstos con los hijos, mientras se realizaba la entrevista ${ }^{2}$. Su investigación tiene como objetivo conocer el impacto de las pautas de conducta familiares y su repercusión en la personalidad del niño. De la combinación de estas variables: control, afecto y comunicación, Baumrind resalta tres estilos educativos paternos, mediante los que los progenitores controlan la conducta de sus hijos: a) "authoritarian discipline" o estilo autoritario; b) "permissive discipline" o estilo no restrictivo, permisivo; y c) un estilo que bautizó como autoritativo, "authoritative discipline". Había considerado que el último estilo tendría mejores resultados que los otros dos. Los resultados obtenidos por Diana Baumnrind, al

2. La metodología utilizada por BAumRind (1971, 239) en el primer estudio realizado (1967) es la siguiente: dividió a los niños en tres tipos de estructura personal según su conducta: a) los niños de la estructura I eran los más competentes, contentos e independientes, confiados en sí mismos y mostraban conductas exploratorias; b) los niños de la estructura II eran medianamente confiados y capaces de controlarse a sí mismos y, en cierto modo, inseguros y temerosos; y c) los niños de la estructura III se manifestaban inmaduros y dependientes, con menos capacidad de control y confianza en sí mismos. Seguidamente, se correlacionó la estructura de la personalidad con los métodos de crianza en la familia y se encontró lo siguiente: a) los padres del grupo I, especialmente las madres, ejercían un control firme, exigencias de ciertos niveles de madurez y buena comunicación con los hijos (se ha llamado comportamiento autoritativo parental); b) los del grupo II eran menos cuidadosos y atentos con sus hijos en comparación con otros grupos (son denominados padres autoritarios); y c) los padres del grupo III eran afectuosos y atentos, pero ejercían poco control y escasas demandas de madurez sobre sus hijos (denominados padres permisivos). En el segundo estudio de 1971, también, los padres autoritativos, al compararlos con los otros modelos estudiados, mostraban más facilidad en el desarrollo de competencias instrumentales en los niños, obteniendo puntuaciones más altas en responsabilidad social e independencia. 
comparar los distintos estilos parentales, confirmaban sus hipótesis y han llegado a ser ampliamente conocidos. Así, los niños de padres autoritativos, comparados con los de padres autoritarios o permisivos, eran más maduros y competentes.

En síntesis, dicha investigación nos demuestra cómo en el marco del hogar en el que se prestan atenciones y cuidados a los niños de edad preescolar y se exigen ciertos niveles de control, se fomenta en los niños madurez y competencia. Por el contrario, estos niveles no se consiguen si se utiliza una disciplina autoritaria, severidad en los castigos o abundantes restricciones y protección excesiva.

Posteriormente, Baumrind, en 1977, estudió la conducta de la misma muestra, cuando los niños tenían 8/9 años de edad y observó que los niños de familias democráticas tenían elevadas competencias sociales y cognitivas; los de progenitores autoritarios se situaban en un nivel medio y los niños de padres permisivos tenían niveles más bajos. Trabajos posteriores han mostrado que, en la adolescencia, se mantenían los mismos efectos.

La descripción de estas tres categorías ha sido ampliamente difundida. Nos limitamos a destacar sus características más significativas con el aviso de que esta tipología representa tendencias más que una clasificación cerrada. En la práctica educativa, las situaciones son más complejas y es difícil encasillar a las familias en una u otra tipología. Por ello, el aspecto más relevante de la tipología, desde nuestro punto de vista, es el análisis de las dimensiones implicadas en las prácticas educativas y cómo son utilizadas.

Los padres autoritarios (authoritarian discipline) valoran la obediencia como una virtud, así como la dedicación a las tareas marcadas, la tradición y la preservación del orden. Favorecen las medidas de castigo o de fuerza y están de acuerdo en mantener a los niños en un papel subordinado y en restringir su autonomía. Dedican muchos esfuerzos a influir, controlar y evaluar el comportamiento y actitudes de sus hijos de acuerdo con unos rígidos patrones preestablecidos. No facilitan el diálogo y, en ocasiones, rechazan a sus hijos/as como medida disciplinaria. El estilo autoritario es el que tiene repercusiones más negativas sobre la socialización de los hijos, como la falta de autonomía personal y creatividad, menor competencia social o baja autoestima y genera niños descontentos, reservados, poco tenaces a la hora de perseguir metas, poco comunicativos y afectuosos y tienden a tener una pobre interiorización de valores morales (MacCoby y Martin, 1983; Dornbusch, Ritter, Leiderman, Roberts y Fraleigh, 1987; Moreno y Cubero, 1990; Baumrind, 1996; Kaufmann, Gesten, Santa Lucia, Salcedo, Rendina-Gobioff y Gadd, 2000; Belsky, Sligo, Jaffee, Woodward y Silva, 2005).

Los padres permisivos (permissive discipline) proporcionan gran autonomía al hijo siempre que no se ponga en peligro su supervivencia física. El prototipo de adulto permisivo requiere que se comporte de una forma afirmativa, aceptadora y benigna hacia los impulsos y las acciones del niño. Su objetivo fundamental es liberarlo del control y evitar el recurso a la autoridad, el uso de las restricciones y castigos. No son exigentes en cuanto a las expectativas de madurez y responsabilidad en la ejecución de las tareas. Uno de los problemas que presenta el estilo permisivo 
consiste en que los padres no siempre son capaces de marcar límites a la permisividad, pudiendo llegar a producir efectos socializadores negativos en los niños respecto a conductas agresivas y el logro de independencia personal. Aparentemente, este tipo de padres forman niños alegres y vitales, pero dependientes, con altos niveles de conducta antisocial y con bajos niveles de madurez y éxito personal (Steinberg, Elmen y Mounts, 1989; Lamborn, Mounts, Steinberg y Dornbusch, 1991; Domínguez y Carton, 1997; Banham, Hanson, Higgins y Jarrett, 2000).

Los padres autoritativos o democráticos (authoritative discipline) intentan dirigir la actividad del niño imponiéndole roles y conductas maduras pero utilizan el razonamiento y la negociación. Los padres de este estilo educativo tienden a dirigir las actividades del niño de forma racional. Parten de una aceptación de los derechos y deberes propios, así como de los derechos y deberes de los niños, lo que la autora consideraba como una "reciprocidad jerárquica", es decir, cada miembro tiene derechos y responsabilidades con respecto al otro. Es un estilo que se caracteriza por la comunicación bidireccional y un énfasis compartido entre la responsabilidad social de las acciones y el desarrollo de la autonomía e independencia en el hijo. Dicho estilo produce, en general, efectos positivos en la socialización: desarrollo de competencias sociales, índices más altos de autoestima y bienestar psicológico, un nivel inferior de conflictos entre padres e hijos, entre otras. Estos niños suelen ser interactivos y hábiles en sus relaciones con sus iguales, independientes y cariñosos (Carter y Welch, 1981; Dornbusch, Ritter, Mont-Reynaud y Chen, 1987; Dornbusch, Ritter, Liederman, Roberts y Fraleigh, 1987; Eisenberg, 1990; Baumnrind, 1991a y b, 1996; Steinberg, 1991; El-Feky, 1991; Lamborn, Mounts, Steinberg y Dornbusch, 1991; Darling y Steinberg, 1993; Domínguez y Carton, 1997; Kaufmann, Gesten, Santa Lucia, Salcedo, Rendina-Gobioff y Gadd, 2000; Banham, Hanson, Higgins y Jarrett, 2000; Chao, 2001; Warash y Markstrom, 2001; García, Pelegrina y Lendínez, 2002; Mansager y Volk, 2004; Gfroerer, Kern y Curlette, 2004; Winsler, Madigan y Aquilino, 2005) . $^{3}$

En la reformulación que MacCoby y Martin (1983) realizaron de las investigaciones de Baumrind, se reinterpretan las dimensiones básicas propuestas por la autora, redefiniéndose los estilos parentales en función de dos aspectos: a) el control o exigencia: presión o número de demandas que los padres ejercen sobre sus hijos para que alcancen determinados objetivos y metas; b) el afecto o sensibilidad

3. En los últimos años se han invertido muchos esfuerzos para elaborar conocimientos y teorías específicas sobre las prácticas educativas familiares. Es conveniente considerar que es un campo de investigación y teorización incipiente pero cuyos frutos parecen ser prometedores. Para revisiones en lengua española ver: Doumanis, 1983; Aznar y Pérez, 1986; Musitu, Román y Gracia, 1988; Moreno y Cubero, 1990; Coloma Medina 1990, 1993 a y b; 1994 a y b; Musitu y Molpereces, 1992; Colom, 1994; Torres, Alvira, Blanco y Sandi, 1994; Palacios y Moreno, 1995; Musitu, Román y Gutiérrez, 1996; Sorribes y García, 1996; Aznar, 1996; Pérez Alonso-Geta y Vidal, 1996; Ceballos y Rodrigo, 1998; Vila, 1998; Solé, 1998; Rodrigo y Palacios, 1998; Gracia Fuster y Musitu, 2000; Musitu y Cava, 2001; Pérez AlonsoGeta, 2001, 2002a y b; Valdivia, 2003; Vázquez, Sarramona y Vera, 2004. 
$y$ calidez: grado de sensibilidad y capacidad de respuesta de los padres ante las necesidades de los hijos, sobre todo, de naturaleza emocional.

Según estos autores, de la combinación de las dimensiones mencionadas y de su grado, se obtienen cuatro estilos educativos paternos: estilo autoritario-recíproco autoritario-represivo, permisivo-indulgente y permisivo-negligente. El estilo permisivo que Baumrind había descrito ha sido dividido por MacCoby y Martin en dos estilos nuevos, al observar que la permisividad presentaba dos formas muy diferentes: el estilo permisivo-indulgente y el permisivo-negligente, este último desconocido en el modelo de Diana Baumrind y que se asocia a un tipo de maltrato (ver Tabla 1).

TABla 1. Estilos educativos Reformulados Por MacCoby y Martin (1983)

\begin{tabular}{|lll|}
\hline & $\begin{array}{l}\text { Reciprocidad. } \\
\text { Implicación afectiva } \\
\text { (Responsiveness) }\end{array}$ & $\begin{array}{l}\text { No reciprocidad. } \\
\text { No implicación afectiva } \\
\text { (Unresponsiveness) }\end{array}$ \\
$\begin{array}{lll}\text { Control fuerte } \\
\text { (Demandingness) }\end{array}$ & AUTORITARIO- & AUTORITARIO- \\
RECÍPROCO & REPRESIVO \\
Control laxo & PERMISIVO- & PERMISIVO- \\
(Undemandingness) & INDULGENTE & NEGLIGENTE \\
\hline
\end{tabular}

Los estilos educativos tienen una gran repercusión y consecuencias evolutivas que no se circunscriben sólo a la etapa infantil, sino que se prolongan a lo largo de la vida. La investigación de Lamborn, Mounts, Steinberg y Dournbusch (1991), realizada a adolescentes entre 14 y 18 años, clasifica a los adolescentes en los cuatro grupos de estilos educativos que definen MacCoby y Martin, contrastando cuatro grupos de resultados en aspectos como el desarrollo psicosocial, el logro escolar, las destrezas interiorizadas y las conductas problemáticas (también son abordadas dichas dimensiones en las investigaciones de Dornbusch, Ritter, Liederman, Roberts y Fraleigh, 1987; Steinberg, Elmen y Mounts, 1989; Steinberg, Lamborn, Darling, Mounts y Dornbusch, 1994; Weiss y Schwarz, 1996). Los resultados indican que los adolescentes que caracterizan a sus padres como autoritativos obtienen puntuaciones más altas en competencias psicosociales y más bajas en medidas de disfunción psicosocial y comportamental. Lo contrario son adolescentes que describen a sus padres como negligentes. Los adolescentes cuyos padres son distinguidos como autoritarios obtienen medidas razonablemente dentro de la obediencia y la conformidad de los adultos, pero tienen un pobre autoconcepto respecto a otros jóvenes. Por lo contrario, adolescentes de hogares indulgentes evidencian un fuerte autoconcepto, pero presentan una frecuencia más alta de abuso 
de sustancias tóxicas y malas conductas escolares, a la vez que son menos comprometidos en la escuela. Dichos resultados apoyan la estructura de MacCoby y Martin e indican la necesidad de distinguir entre dos tipos de familias permisivas: las indulgentes y las negligentes.

El estilo permisivo-indulgente podría definirse por tres características fundamentales de la conducta parental ante el niño: a) la indiferencia ante sus actitudes y conductas tanto positivas como negativas; b) la permisividad y c) la pasividad. Evitan, en lo posible, la afirmación de la autoridad y la imposición de restricciones. Hacen escaso uso de castigos, tolerando todos los impulsos de los hijos. En los hogares, se promueve la comunicación abierta y el clima democrático. Este estilo de disciplina familiar se describe, principalmente, por una interacción carente de sistematización y no suele ofrecer un modelo con el que el hijo pueda identificarse o imitar. Los padres no son directivos, ni asertivos y tampoco establecen normas en la distribución de tareas o en los horarios dentro del hogar. Acceden fácilmente a los deseos de los hijos. Son tolerantes en cuanto a la expresión de impulsos, incluidos los de ira y agresividad, pero debemos destacar que les preocupa la formación de sus hijos. Responden y atienden a sus necesidades, a diferencia de los permisivos-negligentes, cuya implicación y compromiso paterno es nulo. Según los estudios, los hijos de hogares permisivos presentan índices favorables en espontaneidad, originalidad y creatividad, así como mejor competencia social. Se ven favorecidos en autoestima y confianza. Obtienen puntuaciones más bajas en los logros escolares y menor capacidad para la autorresponsabilidad, siendo más propensos a la falta de autocontrol y autodominio. Podemos pensar que la excesiva tolerancia de los padres respecto a los impulsos de los hijos, unida a la tendencia a complacerles, les conduce a no dar valor al esfuerzo personal.

MacCoby y Martin no describen las peculiaridades del estilo permisivo-negligente, por lo que es preciso deducirlas de lo que se dice sobre la implicación paterna. Estos padres se caracterizarían por la no implicación afectiva en los asuntos de los hijos y por la dimisión en la tarea educativa. La permisividad no es debida a razones ideológicas, como ocurre en el estilo permisivo-indulgente, sino a razones pragmáticas, tanto por la falta de tiempo o de interés como por la negligencia o la comodidad. Los padres permisivo-negligentes invierten en los hijos el mínimo tiempo posible y tienden a resolver las obligaciones educativas de la manera más rápida y cómoda posible. Les resulta más cómodo no poner normas, pues éstas implicarían diálogo y vigilancia. No pueden evitar, en algunas ocasiones, estallidos irracionales de ira contra los hijos, cuando éstos traspasan los límites de lo tolerable, debido a su permisividad. Si sus recursos se lo permiten, complacen a los hijos en sus demandas, rodeándolos de halagos materiales. Aunque admiten notables variaciones según de qué familia concreta se trate, es el estilo con efectos socializadores más negativos. Según los estudios, estos niños obtienen las más bajas puntuaciones en autoestima, en desarrollo de capacidades cognitivas y en los logros escolares, así como en la autonomía y en el uso responsable de la libertad. 


\subsection{Otras investigaciones sobre estilos educativos parentales}

Además de analizar en estas páginas las tipologías clásicas de Baumrind y MacCoby y Martin, es de nuestro interés señalar algunas aportaciones, tanto de ámbito nacional como internacional, que han contribuido al estudio de las prácticas educativas paternas, incluyendo nuevos modelos. En el ámbito internacional, cabe presentar el modelo de Hoffman (1970) y la tipología de Kellerhalls y Montandon (1997), además de resaltar la influencia que el libro Inteligencia emocional de Daniel Goleman (1996) ${ }^{4}$ ha ejercido en el tema de las relaciones padres-hijos. En el ámbito nacional, indicamos el estudio de Alberdi (1995), Informe sobre la situación de la familia española, publicado con ocasión del Año Internacional de la Familia, además de las investigaciones de Torres, Alvira, Blanco y Sandi (1994), López Franco (1998) y Ochaita (1995).

Hoffman (1970), cuya clasificación de las estrategias disciplinarias de los padres ha sido muy utilizada, describe los siguientes modelos: afirmación de poder (power assertion) supone el uso de castigos físicos, amenazas verbales, retiradas de privilegios y una gran variedad de técnicas coercitivas (se asemeja al estilo autoritario de Baumrind); retirada de afecto (love withdrawal) utiliza el enfado de los padres y la desaprobación ante las conductas negativas, ignorando al niño sin hablarle ni escucharle; y, la inducción (induction) conlleva connotaciones positivas, ya que, a través de explicaciones de normas, principios, valores y del ofrecimiento de razones para no comportarse mal, trata de "inducir" una motivación intrínseca en el niño. Este último se asemeja al estilo democrático de Baumrind, excepto en que no se utiliza un control-guía para marcar normas y directrices claras. La inducción es el medio de control más indirecto que enfatiza las consecuencias negativas del daño causado a otros, fomentando la empatía hacia éstos. Por el contrario, la afirmación de poder y la retirada de afecto son un medio de control más directo que llevan a una motivación extrínseca, según la cual hay que portarse bien para evitar el castigo.

Para Kellerhalls y Montandon (1997), tres pueden ser los estilos de las familias, que dependen, a su vez, del tipo de interacción que se establece en su seno y de su condición socioeconómica:

a) El estilo contractualista, distinguido por la importancia que los padres dan a la autorregulación y autonomía del niño, así como por el énfasis

4. En la obra, se destaca la importancia del juego como una forma adecuada y positiva de enseñar capacidades relacionadas con la inteligencia emocional y cómo, a través de estudios, ha quedado demostrado que los niños con capacidades altas, en dicho campo, son más felices, confiados y tienen más éxito en la escuela. A lo largo de su obra, padres y educadores encontrarán consejos prácticos y accesibles para enseñar a los hijos a superar sentimientos negativos como la ira y la timidez, la vergüenza y la culpa; alentar la empatía y la emoción; trabajar en grupo; soportar las burlas; motivarse cuando las cosas se ponen difíciles; enseñarles a ser optimistas; enseñar la importancia del humor para minimizar el dolor; ayudarles a percibir el valor del esfuerzo; a administrar el tiempo y el trabajo, etc. 
SUSANA TORÍO LÓPEZ, JOSÉ VICENTE PEÑA CALVO Y M. ${ }^{a}$ DEL CARMEN RODRÍGUEZ MENÉNDEZ

puesto en los valores de la imaginación y creatividad. Desde el punto de vista de las técnicas pedagógicas, este estilo se caracteriza por una escasa insistencia en la obligación o control y pone un mayor énfasis en la incitación, el estímulo o la motivación. Este tipo de familias están abiertas a las influencias del exterior, tales como: el colegio, los amigos, la televisión... Los roles educativos de los padres están poco diferenciados, ambos incluyen aspectos instrumentales y expresivos asumiendo un papel más cercano. Es importante señalar la importancia en este modelo de la variable clase social y de los agentes externos.

b) El estilo estatuario se sitúa en el polo opuesto por la gran importancia que se concede a la obediencia y a la disciplina, al tiempo que implica una menor valoración de la autorregulación y de la sensibilidad del niño. Sus métodos pedagógicos apelan más al control que a la motivación o a la relación. Las distancias entre padres e hijos son considerables, existiendo poca comunicación y escasas actividades comunes. Los roles educativos de los padres están claramente diferenciados y la reserva ante los agentes de socialización del exterior es bastante significativa.

c) El estilo maternalista se caracteriza por la insistencia en la acomodación (obediencia y conformidad) más que en la autonomía o la autodisciplina. Sus técnicas de influencia se basan más en el control que en la motivación o la relación. Existe una gran proximidad entre padres e hijos, se organizan muchas actividades en común, la comunicación entre ellos es estrecha y relativamente íntima, aunque los papeles educativos de los padres tienen perfiles distintos y la apertura a las influencias del exterior es bastante limitada.

López Franco (1998), basándose en los estudios de Gottman y De Claire, habla de cuatro estilos parentales: los padres simplistas, laissez-faire, desaprobadores y los padres verdaderos preparadores emocionales de sus hijos. Resalta la importancia de las interacciones emocionales con los hijos, cuya preparación emocional, ejercida por los padres, influye significativamente en el éxito y felicidad de los hijos.

Torres y colaboradores (1994), Alberdi (1995) y Ochaita (1995), en sus informes e investigaciones, coinciden en describir ampliamente las características formales de una tipología de relación padres e hijos, basada en tres estilos: modelo autoritario, modelo inductivo de apoyo y modelo errático o inconsistente.

En síntesis, el amplio conjunto de investigaciones ha ido mostrando la existencia de una serie de características en las que los padres y las madres difieren unos de otros en sus prácticas educativas. En estas mismas investigaciones se han intentado explicar, en función de tales patrones de actuación, las diferencias interindividuales de los niños y las niñas en sus características de personalidad y socialización. A continuación, presentamos una tabla-resumen de las principales 
tipologías y las pautas familiares e infantiles derivadas de tales contextos (ver Tabla 2$)^{5}$.

TABLA 2. ESTILOS DE EDUCACIÓN FAMILIAR Y COMPORTAMIENTO INFANTIL

\begin{tabular}{|c|c|c|}
\hline $\begin{array}{c}\text { Tipología de socialización } \\
\text { familiar }\end{array}$ & $\begin{array}{l}\text { Rasgos de conducta } \\
\text { parental }\end{array}$ & $\begin{array}{c}\text { Consecuencias educativas } \\
\text { sobre los hijos }\end{array}$ \\
\hline DEMOCRÁTICO & $\begin{array}{l}\text { - Afecto manifiesto } \\
\text { - Sensibilidad ante las } \\
\text { necesidades del niño: } \\
\text { responsabilidad } \\
\text { - Explicaciones } \\
\text { - Promoción de la conducta } \\
\text { deseable } \\
\text { - Disciplina inductiva o téc- } \\
\text { nicas punitivas razonadas } \\
\text { (privaciones, reprimendas) } \\
\text { - Promueven el intercambio } \\
\text { y la comunicación abierta } \\
\text { - Hogar con calor afectivo y } \\
\text { clima democrático }\end{array}$ & $\begin{array}{l}\text { - Competencia social } \\
\text { - Autocontrol } \\
\text { - Motivación } \\
\text { - Iniciativa } \\
\text { - Moral autónoma } \\
\text { - Alta autoestima } \\
\text { - Alegres y espontáneos } \\
\text { - Autoconcepto realista } \\
\text { - Responsabilidad y } \\
\text { fidelidad a compromisos } \\
\text { personales } \\
\text { - Prosociabilidad dentro y } \\
\text { fuera de la casa } \\
\text { - altruismo, solidaridad) } \\
\text { - Elevado motivo de logro } \\
\text { - Disminución en frecuencia } \\
\text { e intensidad de conflictos } \\
\text { padres-hijos }\end{array}$ \\
\hline AUTORITARIO & $\begin{array}{l}\text { - Normas minuciosas y } \\
\text { rígidas } \\
\text { - Recurren a los castigos y } \\
\text { muy poco a las alabanzas } \\
\text { - No responsabilidad } \\
\text { paterna } \\
\text { - Comunicación cerrada o } \\
\text { unidireccional (ausencia } \\
\text { de diálogo) } \\
\text { - Afirmación de poder } \\
\text { - Hogar caracterizado por } \\
\text { un clima autocrático }\end{array}$ & $\begin{array}{l}\text { - Baja autonomía y } \\
\text { autoconfianza } \\
\text { - Baja autonomía personal } \\
\text { y creatividad } \\
\text { - Escasa competencia social } \\
\text { - Agresividad e } \\
\text { impulsividad } \\
\text { - Moral heterónoma } \\
\text { (evitación de castigos) } \\
\text { - Menos alegres y } \\
\text { espontáneos }\end{array}$ \\
\hline
\end{tabular}

5. Esta clasificación es una adaptación de elaboración propia, tomando las aportaciones de Coloma (1993a y 1994b), Rodrigo y Palacios (1998), Vila (1998), Pereira y Pino (2002), junto con la investigación de Lamborn, Mounts, Steinberg y Dournbusch (1991). 
SUSANA TORÍO LÓPEZ, JOSÉ VICENTE PEÑA CALVO Y M. ${ }^{a}$ DEL CARMEN RODRÍGUEZ MENÉNDEZ

\begin{tabular}{|c|c|c|}
\hline NEGLIGENTE & $\begin{array}{l}\text { - Indiferencia ante sus acti- } \\
\text { tudes y conductas tanto } \\
\text { positivas como negativas } \\
\text { - Responden y atienden las } \\
\text { necesidades de los niños } \\
\text { - Permisividad } \\
\text { - Pasividad } \\
\text { - Evitan la afirmación de } \\
\text { autoridad y la imposición } \\
\text { de restricciones } \\
\text { - Escaso uso de castigos, } \\
\text { toleran todos los impulsos } \\
\text { de los niños } \\
\text { - Especial flexibilidad en el } \\
\text { establecimiento de reglas } \\
\text { - Acceden fácilmente a los } \\
\text { deseos de los hijos }\end{array}$ & $\begin{array}{l}\text { - Baja competencia social } \\
\text { - Pobre autocontrol y } \\
\text { heterocontrol } \\
\text { - Escasa motivación } \\
\text { - Escaso respeto a normas } \\
\text { y personas } \\
\text { - Baja autoestima, } \\
\text { inseguridad } \\
\text { - Inestabilidad emocional } \\
\text { - Debilidad en la propia } \\
\text { identidad } \\
\text { - Autoconcepto negativo } \\
\text { - Graves carencias en } \\
\text { autoconfianza y } \\
\text { autorresponsabilidad } \\
\text { - Bajos logros escolares }\end{array}$ \\
\hline INDULGENTE & $\begin{array}{l}\text { - No implicación afectiva } \\
\text { en los asuntos de los hijos } \\
\text { - Dimisión en la tarea } \\
\text { educativa, invierten en } \\
\text { los hijos el menor tiempo } \\
\text { posible } \\
\text { - Escasa motivación y } \\
\text { capacidad de esfuerzo } \\
\text { - Inmadurez } \\
\text { - Alegres y vitales }\end{array}$ & $\begin{array}{l}\text { - Escasa competencia social } \\
\text { - Bajo control de impulsos } \\
\text { y agresividad } \\
\text { - Escasa motivación y } \\
\text { capacidad de esfuerzo } \\
\text { - Inmadurez } \\
\text { - Alegres y vitales }\end{array}$ \\
\hline
\end{tabular}

Se pone en evidencia que el modelo de familia democrática es el más propicio por ser el más educativo para favorecer el desarrollo de la personalidad de los menores y estimular sus capacidades, pautas sociales, habilidades de comunicación y socialización. Exige de los adultos seguridad, serenidad y capacidad de reflexión. Desde nuestra perspectiva, la validez de dicho estilo está en el equilibrio entre el afecto y la autoridad, siendo importante establecer normas y límites.

\section{LA INVESTIGACIÓN ACTUAL SOBRE ESTILOS EDUCATIVOS FAMILIARES}

La investigación longitudinal realizada por Baumrind durante más de tres décadas ha analizado la relación entre tendencias de comportamiento de los padres y la competencia social en los niños de 3 a 15 años. Así mismo, ha revisado la influencia positiva del estilo autoritativo o democrático en variables como el género, la etnia, el estatus socioeconómico, la edad y la estructura familiar. Numerosos estudios posteriores han analizado y corroborado la eficacia de las prácticas 
democráticas, pero pocos han sido los trabajos que han relacionado dichos supuestos con la "Individual Psychology". En esta temática merece especial atención la teoría de Alfred Adler (1927), fundador de esta corriente y que a comienzos de siglo XX había destacado la influencia de la interacción padres e hijos en el desarrollo de la personalidad del niño/a, especialmente, la importancia de la atmósfera familiar y los roles parentales en un óptimo desarrollo y bienestar infantil. Las ideas de Adler han sido elaboradas y extendidas a su muerte, en primer lugar, por Dreikurs (1964, 1992) en Estados Unidos y, en segundo lugar, por otros autores del campo de la "Individual Psychology" (Nelson, 1987; Popkin, 1998, 2002; Ballantine, 2001; Mansager y Volk, 2004; Gfroerer, Kern y Curlette, 2004). Sus investigaciones tratan de establecer comparaciones entre las conclusiones de Baumrind y el modelo Adleriano, y poseen importantes contribuciones en terapia y orientación familiar.

Otras investigaciones han contribuido a avanzar en el estudio de los estilos educativos parentales y han sido muy diversas las variables que se han estudiado (ver Tabla 3). Pasamos a describir someramente estos trabajos.

TABLA 3. InVESTIGACIÓN ACTUAL SOBRE ESTILOS EDUCATIVOS PARENTALES

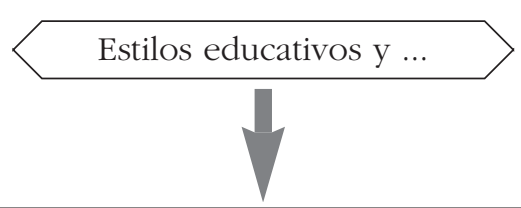

- Éxito en el rendimiento académico

- Autoestima

- Estatus sociométrico de los hijos

- Configuración de un sistema de valores

- Competencia psicosocial

- Niveles de autorrealización en infantes y adolescentes

- Estilos de vida de los adolescentes (hábitos de consumo)

- Comportamiento disocial de los adolescentes

- Estudios comparados entre países

- Técnicas e instrumentos para medición de las prácticas educativas familiares

Una parte de las investigaciones realizadas en este campo, sin pretender ser exhaustivos, están centradas en la relación entre los diferentes estilos educativos parentales y el éxito o competencia académica de los hijos. Las puntuaciones más altas son obtenidas por el alumnado que considera a sus padres como democráticos 
(Dornbusch, Ritter, Mont-Reynaud y Chen, 1987; Steinberg, Elmen y Mounts, 1989; Strassberg, Dodge, Bates y Pettit, 1992; Chao, 1995; Weiss y Schwarz, 1996; Glasgow, Dornbusch, Troyer, Steinberg y Ritter, 1997; Ballantine, 2001; Burchinal, Peisner-Feinberg, Pianta y Howes, 2002; Kim y Rohner, 2002). También en el contexto hispanohablante se ha informado de las relaciones entre el apoyo de los padres y ciertas variables relacionadas con los logros académicos (Martínez, 1987, 1992, 1996; Pérez Díaz, Rodríguez y Sánchez, 2001; Balzano, 2002; Pérez de Pablos, 2003; Peralbo y Fernández, 2003).

La dimensión de afecto por parte de los padres se ha relacionado con el bienestar psicológico y con un nivel óptimo de autoestima en los hijos (Mestre y Frías, 1997; Mayhew y Lempers, 1998; Warash y Markstrom, 2001; García, Pelegrina y Lendínez, 2002; Kim y Chung, 2003; Alonso y Román, 2005).

En numerosos estudios se ha evidenciado que las prácticas de crianza o el estilo de interacción de los padres se relacionan con el estatus sociométrico de sus bijos o la aceptación por parte de sus iguales en preescolar y en la edad escolar (Dishion, 1990; Hart, Ladd y Burleson, 1990; Dekowic y Janssens, 1992; Strassberg, Dodge, Bates y Pettit, 1992; García, Pelegrina y Lendínez, 2002).

De entre todas las instancias de socialización, se analiza la influencia del estilo disciplinar de los padres en la configuración del sistema de valores de sus hijos. (Hoffman, 1975a, 1975b; Eisikovits y Sagi, 1982; Grusec y Goodnow, 1994; Molpereces, Musitu y Lila, 1994; Molpereces, Llinares y Musitu, 2001; López, Bonenberger y Schneider, 2001). La mayor parte de estos estudios sugieren que niños con padres que usan estilos disciplinares inductivos manifiestan niveles más altos de internalización de normas y de desarrollo moral.

En otra línea se sitúan los trabajos que encuentran una relación positiva entre las prácticas educativas paternas y la competencia psicosocial de los hijos que irán adquiriendo en la adolescencia y con las que han de construir un proyecto de vida provechoso y satisfactorio (Lamborn, Mounts, Steinberg y Dornbusch, 1991; Doménech, 1993; Weiss y Schwarz, 1996; Slicker, 1998; Martínez y Fuertes, 1999; Kaufmann, Gesten, Santa Lucia, Salcedo, Rendina-Gobioff y Gadd, 2000; Aunola, Stattin y Nurmi, 2000; Herrera, Brito, Pérez, Martínez y Díaz, 2001; García, Pelegrina y Lendínez, 2002; Oliva, Parra, Sánchez-Queija y López Gaviño, 2007). Incluso se aborda la relación entre los estilos parentales y los niveles de autorrealización en niños y adolescentes (Domínguez y Carton, 1997). Una vez más se pone de manifiesto que el estilo democrático aparece ligado de manera evidente a consecuencias evolutivas más favorables y es el que más apoya el ajuste personal y social de los adolescentes.

En algunas investigaciones se analiza la relación entre los estilos de vida de adolescentes en riesgo -consumo de tabaco, alcohol y otras sustancias, hábitos alimenticios, tenencia de armas, etc.- y la calidad de las relaciones padres-hijos (Weiss y Schwarz, 1996; Pons y Berjano, 1997; Cohen y Rice, 1997; Corvo y Williams, 2000; Rodrigo, Máiquez, García, Mendoza, Rubio, Martínez y Martín, 2004; Orte, 2005, 2006). Las prácticas educativas basadas en la facilidad para establecer 
comunicación y en la expresión de afecto y comprensión, se muestran como factores de protección asociados a los estilos de vida saludables y es percibido el ambiente familiar como un entorno decisivo en la prevención de conductas desajustadas de los adolescentes.

Muchas teorías psicológicas asumen que el comportamiento parental es un determinante en el comportamiento del menor. Diversos estudios abordan la problemática de niños en familias en situación de riesgo social-abuso de alcohol y adicción a drogas, conductas negligentes ante el cuidado de sus hijos, comportamiento criminal parental- y las consecuencias en el comportamiento del menor -exteriorización de problemáticas- (Corvo y Williams, 2000; Thompson, Raynor, Cornah, Stevenson y Sonuga-Barke, 2002; Stanger, Dumenci, Kamon y Burstein, 2004; Parke, Coltrane, Duffy, Buriel, Dennis, Powers, French y Widaman, 2004). Diversos factores demográficos, económicos y psicológicos, determinantes en las prácticas educativas paternas, han sido correlacionados con el comportamiento disocial de los adolescentes. Desde este punto de vista, resaltamos la necesidad de una intervención familiar a través de programas de apoyo y ayuda familiar (a familias con precariedad económica, programas para la prevención de los malos tratos, terapias grupales, líneas telefónicas de apoyo y consulta, programas de educación para padres...), con la finalidad de incrementar la confianza y la competencia de los padres respecto a sus propias habilidades para que puedan fortalecer su rol de padres y cuidadores (mejora de relaciones de comunicación y afecto, adiestramiento en el manejo y utilización de los recursos comunitarios, gestión y organización del hogar, etc.). En estas investigaciones se confirma que la implementación de métodos apropiados y efectivos de compromiso con los padres reduce la violencia escolar y los comportamientos agresivos por parte de los adolescentes.

Un hecho que merece ser destacado (Herrera, Brito, Pérez, Martínez y Díaz, 2001, 47) se refiere a la escasez de investigaciones en las que se consideran los estilos educativos del padre y la madre por separado. Habitualmente se habla de padres democráticos, autoritarios o permisivos de forma genérica, sin tener en cuenta la diferente repercusión que puede tener en los hijos el estilo percibido por cada progenitor. Pero en realidad, las investigaciones han trabajado, fundamentalmente, más con las madres (específicamente, Beckwith y Cohen, 1989; Thompson, Raynor, Cornah, Stevenson y Sonuga-Barke, 2002; Kim y Mahoney, 2004), quizás por el hecho de que habitualmente son éstas las que se encargan de supervisar las costumbres y actividades de la vida cotidiana. Menor investigación existe sobre los estilos educativos de los padres, la correspondencia entre los propios estilos e, incluso, sobre la percepción de los estilos de cada uno de los cónyuges (excepción es el artículo de Winsler, Madigan y Aquilino, 2005). El uso de informaciones múltiples tiene gran relevancia por las implicaciones en la corresponsabilidad que tienen, ambos miembros de la pareja, en las prácticas educativas y en el desarrollo de los hijos. La necesidad de consenso en la utilización de diversas estrategias educativas es una necesidad imperiosa. 
En otro orden de cosas, hemos de mencionar que las familias occidentales han recibido mayor atención por parte de los investigadores, si bien, en esta última década se vienen realizando estudios comparados. La inmigración ha permitido el encuentro de una diversidad de culturas, valores y actitudes y ha contribuido a cambios socioculturales, lo que ha llevado a admitir el estudio de este fenómeno. Se estudian las tendencias de comportamiento de los padres en contextos interculturales, especialmente, padres inmigrantes en Estados Unidos, procedentes de Europa y Asia (Chao, 1995, 2001; Kim y Rohner, 2002; Kim y Chung, 2003), México (Parke, Coltrane, Duffy, Buriel, Dennis, Powers, French y Widaman, 2004) y África (Smetana, 2000). En su mayor parte, los resultados muestran la preferencia y los beneficios de las prácticas educativas democráticas.

La situación descrita ha provocado gran interés en esta temática en el Oriente y ha conducido a la elaboración de estudios en sociedades como Kuwait (El-Feky, 1991) o Hong-Kong y Beijing (Lai, Zhang y Wang, 2000; Shek, 2005), donde se han identificado los modelos de socialización que prevalecen en dichas sociedades, quedando demostrada la posibilidad de aplicar modelos de socialización occidentales en sociedades no-occidentales. De nuevo muestran que el afecto parental, el apoyo y el razonamiento inductivo son asociados con competencia social y mejor rendimiento académico mientras que las prácticas educativas severas predicen problemas de comportamiento en los adolescentes chinos (Chang, Schwartz, Dodge y MacBride-Chang, 2003; Chao, 2001; Kim y Rohner, 2002; Chen, Chang, He y Liu, 2005). Un análisis más detallado perfila diferencias en los estilos parentales de diferentes regiones (Lai, Zhang y Wang, 2000) derivado, en parte, por las diferentes políticas sociales, valores y estilos de vida familiar.

La cultura oriental tradicional ha estado caracterizada por unos roles de género muy definidos donde los padres establecen unos vínculos más estrechos con los hijos y las madres con las hijas, además de observarse diferencias importantes en la interacción educativa: padres estrictos y emocionalmente distantes y madres menos rectas y más afectuosas. Por todo ello, el modelo presentado por Baumnrind como "estilo educativo autoritario" puede ser problemático para los niños inmigrantes de origen asiático por las importantes diferencias culturales. El fuerte control y la obediencia promovida por los padres, con el propósito de mantener la armonía y unidad familiar, explica sus altas puntuaciones en el estilo autoritario. Ante tal situación, se opta por la inclusión de una nueva tipología: "chiao shun o training style" (Chao, 1995) que enfatiza la autodisciplina, la obediencia y el trabajo duro del niño desde edades tempranas.

En la revisión psicopedagógica sobre los estilos de educación familiar cabe mencionar, además, las variadas técnicas o instrumentos elaborados con el propósito de medir las prácticas educativas paternas. De entre éstas, destacamos el Parental Authority Questionnaire de Buri (1991), cuestionario que a través de 48 ítems en forma de escala tipo Likert analiza los modelos disciplinarios ejercitados por el padre y la madre según la tipología propuesta por Baumrind en adolescentes. Otras técnicas que deben señalarse por su novedad son: Parent Behavior 
Checklist (PBC) (Fox, 1994; Brenner y Fox, 1999), que aporta referencias del comportamiento parental en dimensiones como la disciplina, la crianza y expectativas que los niños deben ser capaces de cumplir; The Parent Discipline Scale (PDS) (López, Schneider y Dula, 2002) que mide los efectos de las transgresiones del comportamiento de los infantes en la elección de las intervenciones disciplinarias paternas; Conflict Tactics Scale (CTSPC) utilizado por López, Bonenberger y Schneider (2001) junto con el PDS en el estudio de los estilos disciplinares y el comportamiento moral ${ }^{6}$.

\section{Conclusión}

Como hemos podido comprobar, todos los estudios mencionados hacen hincapié en la influencia de los padres sobre los hijos. Creemos de gran interés insistir en la importancia de las prácticas educativas paternas en un momento en que la estructura familiar está cambiando. Todos los modelos sugieren que los padres son la base para la personalidad del niño y que otorgan un conjunto de funciones psicológicas básicas. De estos modelos se desprende, igualmente, la importancia que tiene la cohesión familiar. Es fundamental que la familia sepa generar en su interior un clima adecuado que satisfaga las necesidades de todos y que se establezca un tipo de interacciones participativas a través del contacto directo. La familia educa a los hijos no sólo directamente por sus intervenciones educativas intencionadas sino, también, indirectamente, por el ambiente en que les hacen crecer.

Somos conscientes de las dificultades que entraña profundizar en el escenario educativo familiar pero es necesario conocer e interpretar las prácticas educativas del momento en el que nos encontramos. Como hemos podido ver los padres disponen de varios modelos o de técnicas disciplinares diversas, pero son las técnicas inductivas de apoyo -o el modelo autoritativo, democrático, contractualista, etc.las más favorecedoras del ajuste social y familiar del niño, así como de proporcionar una adecuada seguridad emocional y autoestima en el mismo. Asegurar un cuidado y sano crecimiento de los hijos, aportar estimulación, ampliar sus relaciones, facilitar un clima de diálogo y de expresividad, encauzar los sentimientos, practicar experiencias de valores, etc., son algunas de las dimensiones básicas que hemos recogido, beneficiarias en el itinerario educativo del niño para alcanzar el correcto desarrollo de su personalidad y una armonía interior. Dicho modelo es por el que

6. Otros instrumentos utilizados en la investigación empírica en el tema que nos ocupa son, entre otros, los siguientes: The Parenting Styles and Dimensions Questionnaire (PSDQ) elaborado por RoBINson, Mandleco, Frost Olsen y Hart (2001); Child Behavior Rating Scale (CBRS) de Mahoney y WheEden (1998) y Maternal Behavior Rating Scale (MBRS) de MAHONEY (1999); The Questionnaire for Parenting Behavior de GERRIS y colaboradores (1993); The Child-Rearing Practices Report (CRPR) de BLOCK (1965); Parents Report de Cohen, Dibble y Grawe (1977) y el Test Autoevaluativo Multifactorial de Adaptación Infantil (TAMAI) de HERNÁNDEZ (1990). 
optamos a la hora de diseñar intervenciones y establecer propuestas para intentar optimizar "las buenas prácticas" y minimizar los factores de riesgo en la vida familiar.

La experiencia nos enseña que no pocos padres y madres están desorientados y dudan de cómo educar a sus hijos. Saben que no pueden repetir las prácticas que observaron en sus progenitores, pero se sienten solos en la tarea de cuidado y crianza. Desearían compartir experiencias y problemas, lo que les conferiría confianza y seguridad en la forma y el modo de educar. En suma, necesitan "aprender a ser padres y madres" adaptados a las exigencias de los tiempos que les ha tocado vivir. En este sentido, entendiendo la familia como escenario de aprendizaje y medio educativo, consideramos necesaria la implementación de programas de educación para la vida familiar. Parece necesario desarrollar una conceptualización más realista sobre las relaciones que sostienen padres/madres e hijos/as para posibilitar una intervención más eficaz en el marco familiar (funciones que cumplen, emociones que se ponen en juego en dichas relaciones, valores que sostienen cada grupo generacional, etc.). En definitiva "cualquier esfuerzo para mejorar la calidad de las relaciones establecidas entre los miembros de la familia contribuirá al desarrollo de un adecuado clima familiar, y este a su vez favorecerá la adaptación de los hijos a dicho contexto" (Triana y Simón, 1994, 274).

Parece difícil anunciar lo que la sociedad del mañana valorará en la tarea de ser padres, pero es importante que éstos sean conscientes del papel que han de desempeñar en relación con la educación de sus hijos, no sólo en cuanto al contenido de su acción educativa, sino en cuanto a las formas o prácticas educativas que rigen dicho proceso. Los niños necesitan dirección y valores, cariño, límites firmes y aceptación.

\section{BibliografíA}

ADleR, A. (1927) Understanding human nature. New York, The World Publishing Company. AlBerdi, I. (1995) Informe sobre la situación de la familia española. Madrid, Ministerio de Asuntos Sociales.

Alonso García, J. y Román Sánchez, J. M. ${ }^{a}$ (2005) Prácticas educativas y autoestima, Psicothema, 17 (1), 76-82.

Aunola, K.; Stattin, H. y Nurmi, J. E. (2000) Parenting styles and adolescents' achievement strategies, Journal of Adolescence, 23 (2), 205-222.

Aznar Minguet, P. (1996) La mediación educativa familiar. Problemática del rol paterno y propuestas educativas, Teoría de la Educación. Revista Interuniversitaria, 8, 129-140.

Aznar Minguet, P. y Pérez Alonso-Geta, P. M. ${ }^{a}$ (1986) La familia y el proceso educativo, en SÁnchez Cerezo, S. (dir.). Enciclopedia de la Educación Preescolar, vol. I. Madrid, Santillana, 435-450.

Ballantine, J. (2001) Raising competent kids: the authoritative parenting style, Childhood Education, 78, 46-47.

Balzano, S. (2002) Las construcciones culturales sobre el éxito y el fracaso escolar y sus implicaciones sobre los modelos educativos en Argentina, Cultura y Educación, 3 (14), 283-296. 
Banham, V.; Hanson, J.; Higgins, A. y Jarrett, M. (2000) Parent-child communication and its perceived effects on the young child's developing self-concept. Paper presented at the Australian Institute of Family Studies Conference. Sydney, Australia.

BAumrind, D. (1966) Effects of authoritative parental control on child behavior, Child Development, 37 (4), 887-907.

- (1967) Child care practices anteceding three patterns of preschool behavior, Genetic Psychology Monographs, 75, 43-88.

- (1968) Authoritarian vs. authoritative control, Adolescence, 3, 255-272.

- (1971) Current patterns of parental authority, Developmental Psychology Monograph, 41 (1), part. 2.

- (1972) An exploratory study of socialization effects on black children: some black-white comparisons, Child Development, 43 (1), 261-267.

- (1978) Parental disciplinary patterns and social competence in children, Youth and Society, 9, 239-276.

- (1991a) Parenting styles and adolescent development, en Lerner, R. M.; Petersen, A. C. y Brooks-Gunn, J. (eds.). Encyclopedia of adolescence, vol. 2. New York, Garland Publishing, 746-758.

- (1991b) The influence of parenting style on adolescent competence and sustance use, Journal of Early Adolescence, 11 (1), 56-95.

- (1996) The discipline controversy revisited, Family relations, 45, 405-414.

- (1997) Necessary distinctions, Psychological Inquiry, 8 (3), 176-229.

Beckwith, L. y Cohen, S. E. (1989) Maternal responsiveness with preterm infants and later competency, New Directions for Child Development, 43, 75-87.

Belsky, J.; Sligo, J.; Jaffee, S. R.; WoOdward, L. y Silva, P. A. (2005) Intergenerational transmission of warm-sensitive-stimulating parenting: a prospective study of mothers and fathers of 3 years olds, Child Development, 76 (2), 384-396.

Block, J. H. (1965) The Child-Rearing Practices Report: a set of $Q$ items for the description of parental socialization attitudes and values. Unpublished manuscript. Berkeley, University of California.

BRENNER, V. y Fox, R. A. (1999) An empirically derived classification of parenting practices, The Journal of Genetic Psychology, 160 (3), 343-356.

Burchinal, M. R.; Peisner-Feinberg, E.; Pianta, R. y Howes, C. (2002) Development of academic skills from preschool through second grade: family and classroom predictors of developmental trajectories, Journal of School Psychology, 40, 415-436.

BuRI, J. R. (1991) Parental Authority Questionnaire, Journal of Personality Assesment, 57 (1), 110-119.

Carter, D. y Welch, D. (1981) Parenting styles and children's behavior, Family relations, 30, 191-195.

Ceballos, E. y Rodrigo, M. ${ }^{a}$ J. (1998) Las metas y estrategias de socialización entre padres e hijos, en Rodrigo, M. ${ }^{a}$ J. y Palacios, J. (coords.). Familia y desarrollo humano. Madrid, Alianza Editorial, 225-243.

Chang, L.; Schwartz, D.; Dodge, K. A. y Macbride-Chang, C. (2003) Harsh parenting in relation to child emotion regulation and aggression, Journal of Family Psychology, 17, 598-606.

CHAO, R. K. (1995) Beyond authoritarianism: a cultural perspective on Asian American parenting practices. Paper presentation for the Symposium "Cultural and contextual influences on parental control. Conference for The American Psychological Association. New York. 
- (2001) Extending research on the consequences of parenting style for Chinese-Americans and European-Americans, Child Development, 72 (6), 1832-1843.

Chen, X.; Chang, L.; He, Y. y Liu, H. (2005) The peer group as a context: moderating effects on relations between maternal parenting and social and school adjustment in Chinese children, Child Development, 76 (2), 417-434.

Cohen, D. A.; Dibble, E. y Grawe, J. (1977) Companion instrument for measuring children's competence and parental style, Archives of Genetic Psychiatry, 30, 805-815.

Cohen, D. A. y Rice, J. (1997) Parenting styles, adolescent substance use, and academic achievement, Journal of Drug Education, 27, 199-211.

Colom Canellas, A. J. (1994) La familia como agencia educativa, en Castillejo, J. L.; VÁzQuez, G.; Colom, A. J. y Sarramona, J. Teoría de la educación. Madrid, Taurus, 227-251.

Coloma Medina, J. (1990) La familia como agencia de socialización, en Fermoso Estébanez, P.; Coloma Medina, J.; Rodríguez Neira, T. y Samper, L. Sociología de la educación. Barcelona, Alamex, 171-192.

- (1993a) La familia como ámbito de socialización de los hijos, en Quintana Cabanas, J. M. ${ }^{\text {a }}$ (coord.). Pedagogía familiar. Madrid, Narcea, 31-43.

- (1993b) Estilos educativos paternos, en Quintana Cabanas, J. M. ${ }^{\text {a }}$ (coord.). Pedagogía familiar. Madrid, Narcea, 45-58.

- (1994a) Socialización familiar. Los estilos educativos paternos y su repercusión en la socialización de los hijos, Teología Espiritual, XXXVIII (113), 257-288.

- (1994b) Los fines de la acción educativa en el ámbito familiar, Escritos de Vedat, 24, 143-163.

Corvo, K. y Williams, K. (2000) Substance abuse, parenting styles and agression: an exploratory study of weapon carrying students, Education, 46 (1), 1-13.

Darling, N. y Steinberg, L. (1993) Parenting style as context: an integrative model, Psychological Bulletin, 114, 487-796.

Dekowic, M. y Janssens, J. M. A. M. (1992) Parents' child-rearing style and child's sociometric status, Developmental Psychology, 28, 925-932.

Dishion, T. (1990) The family ecology of boys' peer relations in middle childhood, Child Development, 61 (3), 874-892.

DOMÉNECH Llaberia, E. (1993) La interacción padres-hijos y sus consecuencias psicopatológicas y psicoterapéuticas, Revista Española de Pedagogía, 196, 531-549.

Domínguez, M. M. y Carton, J. S. (1997) The relationship between self-actualization and parenting style, Journal of Social Behavior and Personality, 12 (4), 1093-1100.

Dornbusch, S. M.; RitTer, P. L.; Liederman, P. H.; Roberts, D. F. y Fraleigh, M. J. (1987) The relation of parenting style to adolescent school performance, Child Development, 58 (2), 1244-1257.

Dornbusch, S. M.; Ritter, P. L.; Mont-Reynaud, R. y Chen, Z. (1987) Family decision making and academic performance in a diverse high school population, Journal of Adolescent Research, 5, 143-160.

Doumanis, M. (1983) Prácticas educativas maternas en entornos rurales y urbanos. Madrid, Visor/MEC.

Dreikurs, R. (1992) The challenge of parenthood. New York, Plume (Original work published 1948).

Dreikurs, R. y Soltz, V. (1964) Children: the challenge. New York, Hawthorn. 
Eisenberg, N. (1990) Prosocial development in early and midadolescence, en Montemayor, R.; ADAMS, G. y Gullota, T. (eds.). From childhood to adolescence: a transitional period? Newbury Park, Sage, 240-268.

Eisikovits, Z. y SAGI, A. (1982) Moral development and discipline encounter in delinquent and nondelinquent adolescent, Journal of Youth and Adolescence, 11, 3, 217-230.

EL-FeKY, H. A. (1991) Patterns of parental control in Kuwaiti society, International Journal of Psychology, 26 (4), 485-495.

ERIKSOn, E. H. (1963) Childhood and society. New York, Norton.

FlaQUER, L. (1993) La socialización en la familia: teorías, modelos e interacciones, en GARCía de león, M. a A.; De la Fuente, G. y Ortega, F. (eds.). Sociología de la educación. Barcelona, Barcanova, 45-69.

Fox, R. A. (1994) Parent Behavior Checklist manual. Austin, TX, Pro-Ed.

Gadeyne, E.; Ghesquiere, P. y Onghena, P. (2004) Longitudinal relations between parenting and child adjustment in young children, Journal of Clinical Child and Adolescent Psychology, 33 (2), 347-358.

García linares, M. C. C.; Pelegrina, S. y Lendínez, J. (2002) Los estilos educativos de los padres y la competencia psicosocial de los adolescentes, Anuario de Psicología, 33 (1), 79-95.

Gerris, J. R. M.; Van Boxtel, D. A. A. M.; Vermulst, A. A.; Janssens, J. M. A. M.; Van Zutphen, R. A. H. y Felding, A. J. A. (1993) Child-rearing and family in the Netherlands. Documentation of an national representative survey of child-rearing, family relations, and family processes in 1990. Nijmegen, The Netherlands, Institute of Family Studies, University of Nijmegen.

Gfroerer, K. P.; Kern, R. M. y Curlette, W. L. (2004) Research support for individual psychology's parenting model, Journal of Individual Psychology, 60 (4), 379-388.

Glasgow, K. L.; Dornbusch, S. M.; Troyer, L.; Steinberg, L. y Ritter, P. L. (1997) Parenting styles, adolescent's attribution and educational outcomes in nine heterogeneous high schools, Child Development, 68 (3), 507-529.

Goleman, D. (1996) Inteligencia emocional. Barcelona, Kairós.

Goodnow, J. J. (1985) Change and variation in ideas about childhood and parenting, en SigeL, I. E. (ed.). Parental belief systems: The psychological consequences for children. Hillsdale, Erlbaum, 235-270.

Gracia Fuster, E. y Musitu OchoA, G. (2000) Psicología social de la familia. Barcelona, Paidós.

Grusec, J. E. y GoOdNow, J. J. (1994) Impact of parental discipline methods on the child's internalization of values: a reconceptualization of current view points, Developmental Psychology, 30, 4-19.

Hart, C. H.; LADD, G. W. y Burleson, B. R. (1990) Children's expectations of the outcomes of social strategies: relations with sociometric status and maternal disciplinary styles, Child Development, 61 (1), 127-137.

Hernández, P. (1990) Test Autoevaluativo Multifactorial de Adaptación Infantil (TAMAI). Madrid, TEA.

Herrera Gutiérrez, E.; Brito de la Nuez, A. G.; Pérez lópez, J.; Martínez Fuentes, M. ${ }^{a}$ T. y Díaz Navarro, A. (2001) Percepción de estilos educativos parentales e inadaptación en adolescentes, Revista de Psicología Universitas Tarraconensis, 23 (1-2), 44-57.

Hoffman, M. L. (1970) Conscience, personality and socialization techniques, Human Development, 13, 90-126. 
- (1975a) Moral internalization, parent power, and the nature of parent-child interaction, Developmental Psychology, 11, 228-239.

- (1975b) Altruistic behavior and the parent-child relationship, Journal of Personality and Social Development, 31, 937-943.

Kaufmann, D.; Gesten, E.; Santa Lucia, R. C.; Salcedo, O.; Rendina-Gobioff, G. y Gadd, R. (2000) The relationship between parenting style and children's adjustment: the parent's perspective, Journal of Child and family studies, 8 (2), 231-245.

Kellerhalls, J. y Montandon, C. (1997) Les styles éducatifs, en De Singly, F. (dir.). La famille l'état des savoirs. Paris, Éditions La Découverte, 194-200.

Kim, H. y Chung, R. H. G. (2003) Relationship of recalled parenting style to self-perception in Korean American College students, Journal of Genetic Psychology, 164 (4), 481-492.

Kim, J. y Mahoney, G. (2004) The effects of mother's style of interaction on children's engagement. Implications for using responsive interventions with parents, Topics in Early Childhood Special Education (TECSE), 24 (1), 31-38.

Kim, K. y Rohner, R. P. (2002) Parental warmth, control and involvement in schooling. Predicting academic achievement among Korean American adolescents, Journal of Crosscultural Psychology, 33 (2), 127-140.

LaI, A. C.; Zhang, Z. y Wang, W. (2000) Maternal child-rearing practices in Hong Kong and Beijing chinese families: a comparative study, International Journal of Psychology, 35 (1), 60-66.

Lamborn, S. D.; Mounts, N. S.; Steinberg, L. y Dornbusch, S. M. (1991) Patterns of competence and adjustment among adolescents from authoritative, authoritarian, indulgent and neglectful families, Child Development, 62 (5), 1049-1065.

López, N. L.; Bonenberger, J. L. y Schneider, H. G. (2001) Parental disciplinary history, current levels of empathy and moral reasoning in young adults, North American Journal of Psychology, 3 (1), 193-204.

López, N. L.; Schneider, H. G. y Dula, C. S. (2002) Parent Discipline Scale: Discipline choice as a function of transgression type, North American Journal of Psychology, 4 (2), 381-394.

López Franco, E. (1998) La familia, nuevo contexto educativo ante el conflicto y la esperanza, Revista Complutense de Educación, 9 (2), 79-100.

MacCoby, E. E. y Martin, J. A. (1983) Socialization in the context of the family: parent-child interaction, en Hetherington, E. M. y Mussen, P. H. (eds.). Handbook of child psychology: vol. 4. Socialization, personality and social development. New York, Wiley, 1-101.

Mahoney, G. (1999) The Maternal Behavior Rating Scale-Revided. (Available from the author, Mandel School of Applied Social Sciences, 11235 Bellflower Rd., Cleveland, OH 441067164).

Mahoney, G. y Wheeden, C. A. (1998) Effects of teacher style on the engagement of preschool aged children with special learning needs, Journal of Developmental and Learning Disorders, 2, 293-315.

Mansager, E. y Volk, R. (2004) Parent's prism: three dimensions of effective parenting, Journal of Individual Psychology, 60 (3), 277-293.

Martínez, J. L. y Fuertes, A. (1999) Importancia del clima familiar y la experiencia de pareja en las relaciones de amistad de los adolescentes, Revista de Psicología Social, 14, 235-250.

MarTínez González, R. A. (1987) Clima afectivo y rendimiento escolar, Aula abierta, 49, 79-94.

- (1992) Factores familiares que intervienen en el progreso académico de los alumnos, Aula abierta, 60, 23-40. 
- (1996) Familia y educación. Fundamentos teóricos y metodológicos. Oviedo, Servicio de Publicaciones de la Universidad de Oviedo.

MAYHEW, K. P. y LEMPERS, J. D. (1998) The relations among financial strain, parenting, parent self-esteem, and adolescent self-esteem, Journal of Early Adolescence, 18, 145-172.

Mestre Escrivá, V. y Frías Navarro, D. (1997) Estilos educativos paternos y desarrollo del autoconcepto en los hijos, Surgam, 449, 17-35.

Molpereces Pastor, M. ${ }^{a}$ A.; Luinares Insa, L. I. y Musitu OchoA, G. (2001) Estilos de disciplina familiar y prioridades de valor en la adolescencia, Revista de Psicología Social Aplicada, 11 (3), 49-67.

Molpereces Pastor, M. ${ }^{a}$ A.; Musitu Ochoa, G. y Lila, M. S. (1994) La socialización del sistema de valores en el ámbito familiar, en Musitu, G. y Allat, P. (eds.). Psicosociología de la familia. Valencia, Albatros, 121-146.

Moreno, M. ${ }^{a}$ C. y Cubero, R. (1990) Relaciones sociales: familia, escuela, compañeros. Años preescolares, en Palacios, J.; Marchesi, A. y Coll, C. (comps.). Desarrollo psicológico y educación I. Psicología Evolutiva. Madrid, Alianza Editorial, 219-232.

Musitu Ochoa, G. y Cava, M. ${ }^{a}$ J. (2001) La familia y la educación. Barcelona, Octaedro.

Musitu OchoA, G. y Molpereces, M. A. (1992) Estilos de socialización, familismo y valores, Infancia y juventud, 16, 11-67.

Musitu Ochoa, G.; Román SÁnchez, J. M. ${ }^{a}$ y Gracia Fuster, E. (1988) Familia y educación. Prácticas educativas de los padres y socialización de los hijos. Barcelona, Labor Universitaria.

Musitu Ochoa, G. ; Román SAnchez, J. M. a y Gutiérrez Sanmartín, M. (1996) Educación familiar y socialización de los hijos. Barcelona, Idea Books.

Nelson, J. (1987) Positive discipline. New York, Ballantine Books.

Ochaita, E. (1995) Desarrollo de las relaciones padres/hijos, Infancia y Sociedad, 30, 206-227.

Oliva Delgado, A.; Parra Jiménez, A.; Sánchez-Queija, I. y López Gaviño, F. (2007) Estilos educativos materno y paterno: evaluación y relación con el ajuste del adolescente, Anales de Psicología, 23 (1), 49-56.

Orte Socías, C. y Gifes (2005) Los programas de prevención de drogas centrados en la familia. Una visión desde la investigación práctica, Proyecto Hombre: Revista de la Asociación Proyecto Hombre, 53, 14-17.

- (2006) Volver a ser una familia después de la droga: aplicación de un programa para reforzar la competencia familiar, Proyecto Hombre: Revista de la Asociación Proyecto Hombre, 57, 43-46.

Palacios, J. y Moreno, M. C. (1995) Contexto familiar y desarrollo social, en Rodrigo, M. ${ }^{a}$ J. (ed.). Contexto y desarrollo social. Madrid, Síntesis Psicología, 157-188.

Parke, R. D.; Coltrane, S.; Duffy, S.; Buriel, R.; Dennis, J.; Powers, J.; French, S. y Widaman, K. F. (2004) Economic stress, parenting and child adjustment in Mexican American and European American families, Child Development, 75 (6), 1632-1656.

Peralbo Uzquiano, M. y Fernández Amado, M. ${ }^{a}$ L. (2003) Estructura familiar y rendimiento escolar en Educación Secundaria Obligatoria, Revista Galego-Portuguesa de Psicoloxía e Educación, 7 (8), 309-322.

Pereira Domínguez, M. ${ }^{a}$ C. y Pino Juste, M. R. (2002) Un programa de intervención pedagógica sobre educación familiar en el ámbito comunitario. Aspectos generales (I), Revista de Ciencias de la Educación, 189, 21-37.

Pérez Alonso-GeTA, P. M. ${ }^{a}$ y Vidal Lucena, M. (dirs.) (1996) Valores y pautas de crianza familiar. El niño de O-6. Estudio interdisciplinar. Madrid, SM. Fundación Santa María. 
SUSANA TORÍO LÓPEZ, JOSÉ VICENTE PEÑA CALVO Y M. ${ }^{a}$ DEL CARMEN RODRÍGUEZ MENÉNDEZ

ESTILOS EDUCATIVOS PARENTALES. REVISIÓN BIBLIOGRÁFICA Y REFORMULACIÓN TEÓRICA

- (2001) Valores y pautas de la crianza familiar en los montes del Pas, Teoría de la Educación. Revista Interuniversitaria, 13, 115-136.

- (2002a) Valores y pautas de interacción familiar en la adolescencia (13-18 años). Madrid, SM. Fundación Santa María.

- (2002b) Crianza y estilos de educación familiar, en Gervilla, E. (coord.). Educación familiar. Nuevas relaciones humanas y humanizadoras. Madrid, Narcea, 65-80.

Pérez de Pablos, S. (2003) El papel de los padres en el éxito escolar de los hijos. Madrid, Santillana.

Pérez Díaz, V.; Rodríguez, C. y SÁnchez, L. (2001) La familia española ante la educación de los hijos. Barcelona, Fundación "la Caixa" (http://www.estudios.lacaixa.es).

Pons Díez, J. y Berjano Peirats, E. (1997) Análisis de los estilos parentales de socialización asociados al abuso de alcohol en adolescentes, Psicothema, 9 (3), 609-617.

Popkin, M. H. (1998) Active parenting of teens. Atlanta, GA: Active Parenting Publishers.

- (2002) Active parenting now. Atlanta, GA: Active Parenting Publishers.

Rich Harris, J. (2002) El mito de la educación. Barcelona, Grupo Editorial Random House Mondadori.

Robinson, C. C.; Mandleco, B.; Frost Olsen, S. y Hart, C. H. (2001) The parenting styles and dimensions questionnaire (PSDQ), en Perlmutter, B. F.; Touliatos, J. y Holden, G. W. (eds.). Handbook of family measurement techniques. Vol. 2: Instruments and index. Thousand Oaks, CA: Sage.

Rodrigo, M. ${ }^{a}$ J.; Máiquez, M. ${ }^{a}$ L.; García, M.; Mendoza, R.; Rubio, A.; Martínez, A. y Martín, J. C. (2004) Relaciones padres-hijos y estilos de vida en la adolescencia, Psicothema, 16 (2), 203-210.

Rodrigo, M. a J. y Palacios, J. (coords.) (1998) Familia y desarrollo bumano. Madrid, Alianza Editorial.

Rodríguez NeIRA, T. (1999) Lo privado. Aprendizajes tácitos, Teoría de la Educación. Revista Interuniversitaria, 11, 85-100.

Rollins, B. C. y Thomas, D. L. (1979) Parental support, power and control techniques in the socialization of children, en BURR, E. R. et al. (eds.). Contemporary theories about the family. New York, Free Press, 317-364.

Schwarz, J. C.; Barton-Henry, M. L. y PruZinsky, Th. (1985) Assesing child-rearing behaviors: a comparison of ratings made by mother, father, child and sibling on the CRPBI, Child Development, 56 (2), 462-479.

SHEK, D. T. L. (2005) Paternal and maternal influences on the psychological well-being, substance abuse, and delinquency of Chinese adolescents experiencing economic disadvantage, Journal of Clinical Psychology, 61 (3), 219-234.

SLICKER, E. K. (1998) Relationship of parenting style to behavioral adjustment in graduating high school seniors, Journal of Youth and Adolescent, 27, 345-372.

Smetana, J. G. (2000) Middle-class African American adolescents' conceptions of parental authority and parenting practices: a longitudinal investigation, Child Development, 71 (6), 1672-1686.

Solé i Gallart, I. (1998) Las prácticas educativas como contextos de desarrollo, en Coll, C. (coord.). Psicología de la educación. Barcelona, Edhasa, 137-216.

Sorribes, S. y García, F. J. (1996) Los estilos disciplinarios paternos, en Clemente, R. A. y Hernández, C. (eds.). Contextos de desarrollo psicológico y educación. Málaga, Aljibe, 151-170. 
Stanger, C.; Dumenci, L.; Kamon, J. y Burstein, M. (2004) Parenting and children's externalizing problems in substance-abusing families, Journal of Clinical Child and Adolescent Psychology, 33 (3), 590-600.

Steinberg, L. (1991) Parent-adolescent relations, en Lerner, R. M.; Petersen, A. C. y BrooksGunn, J. (eds.). Encyclopedia of adolescence, vol. 2. New York, Garland Publishing, 724728.

Steinberg, L.; Elmen, J. D. y Mounts, N. S. (1989) Authoritative parenting, psychosocial maturity, and academic success among adolescents, Child Development, 60 (6), 1424-1436.

Steinberg, L.; Lamborn, S. D.; Darling, N.; Mounts, N. S. y Dornbusch, S. M. (1994) Overtime changes in adjustment and competence among adolescent from authoritative, authoritarian, indulgent, and neglectful families, Child Development, 65 (3), 754-770.

Strassberg, Z.; Dodge, K. A.; Bates, J. E. y Pettit, G. S. (1992) The longitudinal relation between parental conflict strategies and children's sociometric standing in kindergarten, Merrill-Palmer Quarterly, 38, 477-493.

Thomas, D. L.; Gecas, V.; Weigert, A. y RoOney, E. (1974) Family socialization and the adolescent. Lexington, Health Lexington.

Thompson, M. J.; Raynor, A.; Cornah, D.; Stevenson, J. y Sonuga-Barke, E. J. S. (2002) Parenting behaviour described by mothers in a general population sample, Child: Care, Health \& Development, 28 (2), 149-155.

Torres Rius, M.; Alvira Martin, F.; Blanco Moreno, F. y Sandi Pérez, M. (1994) Relaciones padres/hijos. Madrid, Ministerio de Asuntos Sociales.

Triana, B. y Simón, M. ${ }^{a}$ I. (1994) La familia vista por los hijos, en Rodrigo, M. ${ }^{a}$ J. (ed.). Contexto y desarrollo social. Madrid, Síntesis, 271-303.

VAldivia SÁnchez, M. ${ }^{a}$ C. (2003) Los estilos educativos en la educación familiar, Letras de Deusto, 33 (99), 29-62.

Vázquez Gómez, G.; Sarramona lópez, J. y Vera Vila, J. (2004) Familia, educación y desarrollo cognitivo, en SANTOS Rego, M. A. y Touriñán López, J. M. (eds.). Familia, educación y sociedad civil. XXII Seminario Interuniversitario de Teoría de la Educación. Santiago de Compostela, Universidad de Santiago de Compostela, 29-87.

VILA, I. (1998) Familia, escuela y comunidad. Barcelona, ICE Universidad de Barcelona-Horsori.

Warash, B. G. y Markstrom, C. A. (2001) Parental perceptions of parenting styles in relation to academic self-esteem of preschoolers, Education, 121 (3), 485-493.

Weiss, L. H. y SCHWARZ, J. C. (1996) The relationship between parenting types and older adolescents' personality, academic achievement, adjustment and substance use, Child Development, 67 (5), 2101-2114.

Winsler, A.; Madigan, A. L. y Aquilino, S. A. (2005) Correspondence between maternal and paternal parenting styles in early childhood, Early Childhood Research Quarterly, 20, 1-12. 